\title{
Identifying the position of the right atrium to align pressure transducer for CVP
}

\author{
Avellan S \\ Department of Anaesthesiology \& Intensive Care, Sahlgrenska University \\ Hospital, Gothenburg, Sweden \\ Sondergaard S \\ Centre of Elective Surgery, Silkeborg Regional Hospital, Denmark
}

UNIVERSITY OF

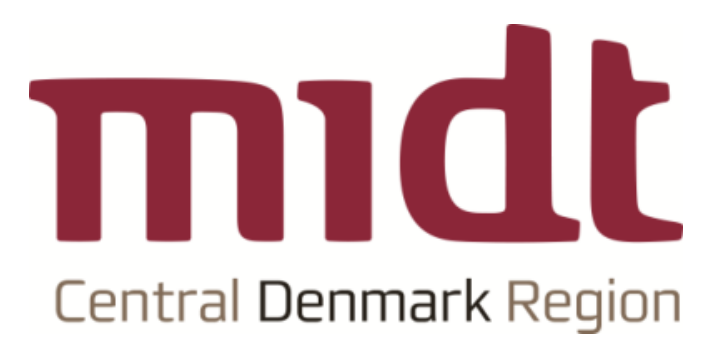

Central Venous Pressure (CVP) has an indisputable role in haemodynamic
management perioperatively and in the intensive care. This becomes clear in the
combined Starling 'cardiac function curve' and Guyton 'venous return curve'. CVP
acts as part of the distending pressure eliciting the Starling 'Law of the Heart' and as
backpressure to venous return. In advanced clinical decision support systems CVP is
fundamental to the computation of the mean systemic filling pressure $\left(P_{\text {msa }}\right)$, the
efficiency of the heart $\left(E_{h}\right)$ and of infused volume $\left(E_{\text {vol }}\right)$, and it is a major determinant
in the control of bleeding in impeding the return of blood from surgically severed
venous plexuses and impairing organ function through stasis. These physiological
relations have been utilized in the Low CVP anaesthesia technique in liver surgery
(and apply to spine surgery, gynaecological, prostate and thoracic surgery as well)
and are documented in the linear relationship between rising CVP and increasing
incidence of acute kidney failure in the ICU.

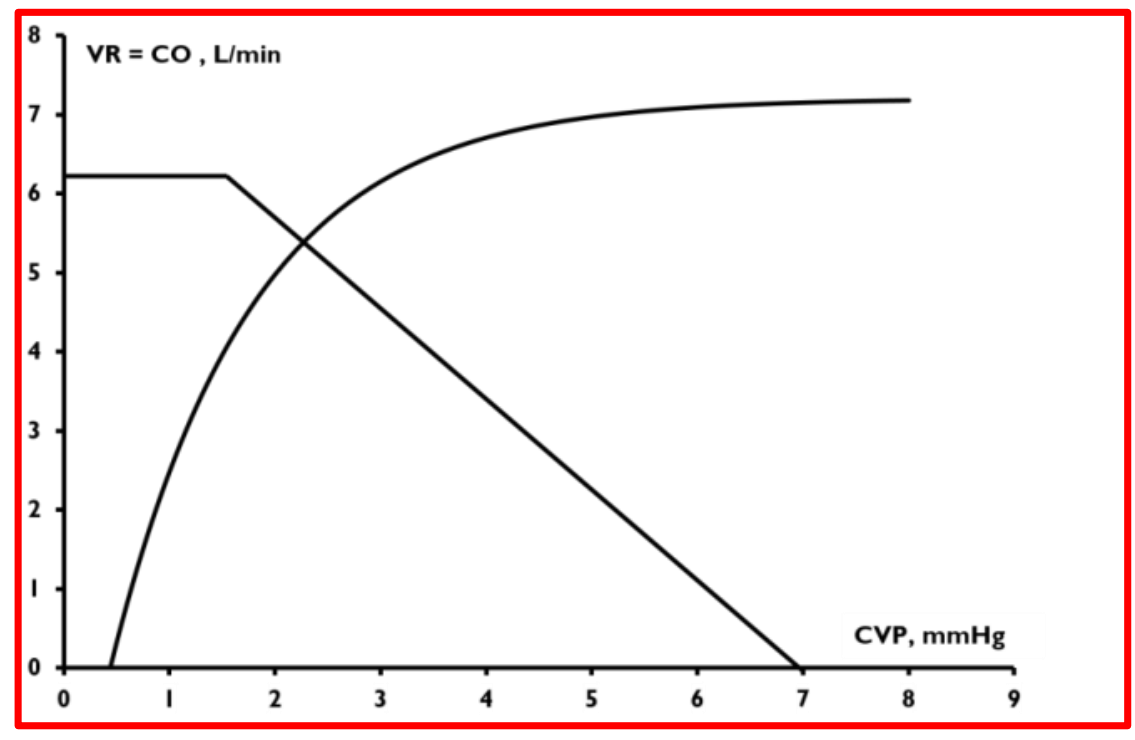

The cardiac function and venous return curves. The working point of the heart is at the intersection of the two curves.

To honour these obvious consequences of the functional organization of the circulation, the clinician must master the correct measurement of the CVP. Traditionally, the pressure sensor is levelled towards the midaxillary line using a spirit level (SL). The projection of the position the tricuspid valve (TV), however, has been shown to be at the cross-point of the coronal midline, at $40-45 \%$ of the anteroposterior distance and the sagittal plane at IC4.

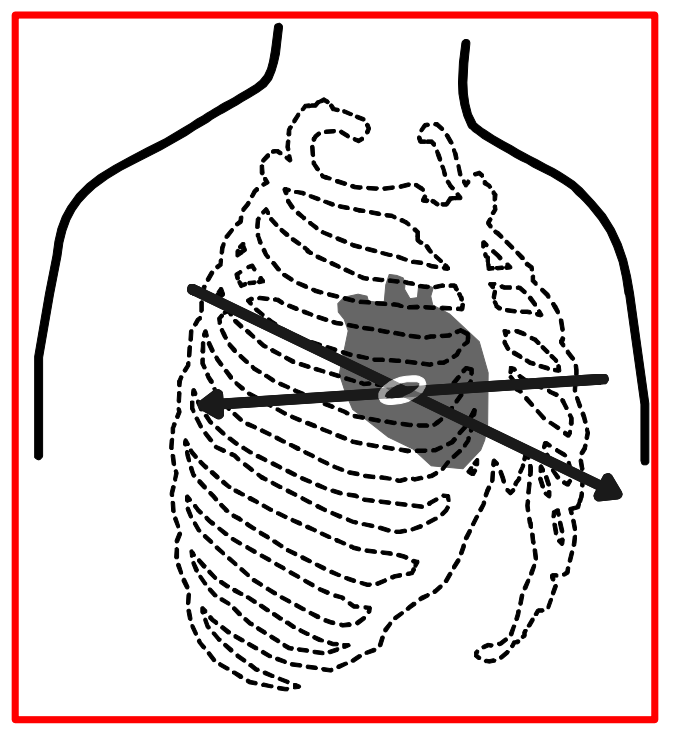

The 3D position of the tricuspid valve in the thorax is projected onto the surface in 1) midline, 2) $40-45 \%$ of AP diameter and 3) at IC4. The TV is regarded as a pivotal point of circulation: As right ventricular pressure exceeds CVP during systole, TV closes and blood is expelled into the pulmonary circulation.

We conducted a study assessing the agreement between the positioning of the pressure sensor by staff in the postoperative ward determining the level by 1) bilateral electromagnetic (EM) sensors at external landmarks and 2) an internal EM sensor in a custom made central venous line with a blinded lumen[1]. In the postoperative ward the patient was placed in a bed where one foot of Styrofoam insulated the EM measurements from the magnetic bedframe. The EM generator was inserted in a cut hole in the mattress under the chest of the patient creating an EM dome around the thorax allowing the detection of the EM sensors. Patients were cared for in supine, tilted and rolled positions. 10-12 measurements were performed by members of staff in 20 patients having undergone major abdominal surgery.

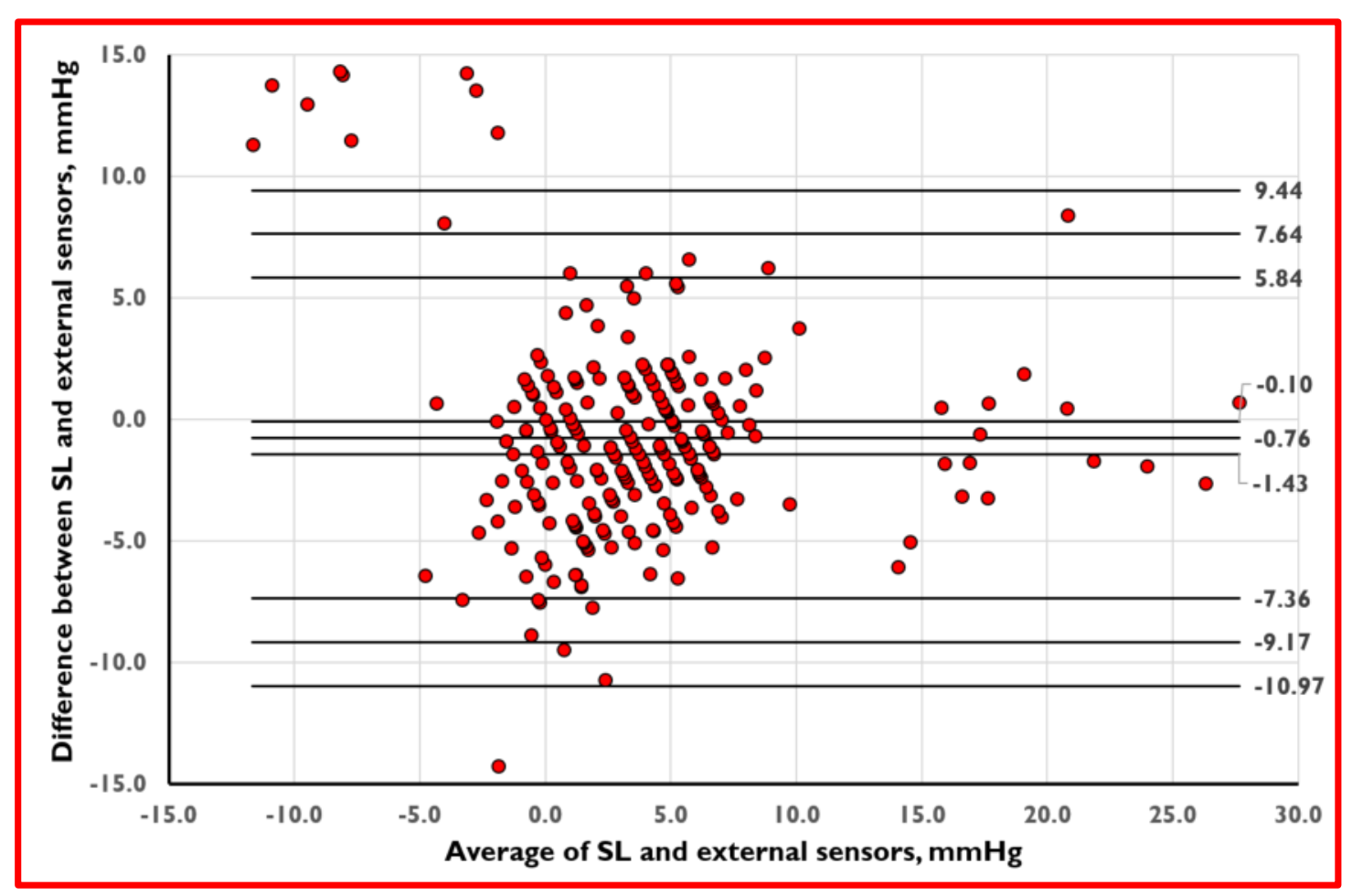

Results demonstrated a clinically unacceptable limits of agreement (LOA) between measurements performed by the EM method (external, based on anatomical landmarks, and internal sensor in CVC) and measurements by using a spirit level. The Bland \& Altman analysis using repeated measurements in the 20 subjects arrived at LOAs close to $\pm 10 \mathrm{mmHg}$. Tilt affected the precision with both external $(p=0.0275)$ and internal sensor $(p<0.0001)$, whereas roll had no influence on precision ( $p=0.085$ and $p=0.289$, respectively).

Bland \& Altman assessment of agreement between EM and SL using repeated measurements of CVP in patients, according to Olofsen[2]. Bias and limits of agreement are displayed with $95 \% \mathrm{Cl}$.

\section{In conclusion, these LOAs are incompatible with the safe conduct of Low CVP anaesthesia and safe cardiovascular regulation in the ICU or postoperative care in high risk surgery. We'll need the EM-based approach to CVP measurement.}

For further information, contact

S. Sondergaard, e-mail: soeresen@rm.dk
1. David Wilkes, Vygon, Ecouen, France is warmly acknowledged for developing and providing the multilumen CVCs with one lumen blinded.

2. Olofsen et al. Improvements in the application and reporting of advanced BlandAltman methods of comparison. JCMC 29 (1):127-139. 2015 